\title{
Effect of process parameters on the volume of the stub bar of Rolled Piece by Cross Wedge Rolling under the different end shapes
}

\author{
Duanlu Guo ${ }^{1, a, *}$, Xin $\mathrm{Lu}^{1}$ \\ ${ }^{1 .}$ China Academy of Machinery Science \& Technology, No. 2, Shouti South Road, Haidian District, \\ 100044 Beijing, China \\ aguoduanlu@outlook.com \\ *Corresponding author
}

Keywords: Cross Wedge Rolling, Stub Bar, Process Parameters, Numerical Simulation.

\begin{abstract}
The forming process of rolling workpieces with different end shapes with cross wedge rolling was simulated by the software Deform-3D. And combined with the principle of blank compensation method, the influence of process parameters on the volume of the stub bar of workpiece with flat end and circular-arc end was analyzed, and the method to effectively control the concavity of rolled piece end surface is brought forward by using circular-arc billet. According to the obtained research results, the volume of stub bar is in direct proportion to the spreading angle, and decreases at first and then increases with the increasing of the forming angle, and increases at first and then decreases with increasing of the area reduction, and increases at first and then keep approximately invariant with increasing of mill length. Finally, the feasibility of using circular-arc billet to reduce the concavity were verified by rolling experiment.
\end{abstract}

\section{Introduction}

Cross wedge rolling (CWR) is a near-net-shape forming process with higher productivity and material utilization, resource saving, better product quality, automation and lower costs. After nearly 50 years of development, CWR process from technology to equipment is gradually mature under the theoretical research and technical development by numerous scholars and engineering technicians at home and abroad. But in theoretical research on less or no material head of CWR is not particularly adult.

Saving material is the permanent theme of the development and research of plastic forming process ${ }^{[1,2]}$. The loss of stub bars has been the unfavorable factor of further popularization and application of cross wedge rolling technology. If we can break through in cross wedge rolling without concavity, that will provide new technical support for popularization and application of cross wedge rolling technology. 
In this paper, the impact of process parameters on the volume of the stub bar of workpiece with flat end and circular-arc end was analyzed, by comparison, the method to effectively control the concavity of rolled piece end surface is brought forward by using circular-arc billet. In the end, the feasibility of using taper billet to reduce the concavity were verified by rolling experiment.

\section{Finite element simulation}

\subsection{The establishment of FEM}

The influence of the process parameters on the stub bar were investigated using a single wedge model. The simulation for the shafts is symmetric in this paper, in order to save simulation time, it can take half of the rolled pieces to simulate. The geometric model of the roller, blank and guide plates were built in UG and saved in STL format. Afterwards, they were imported to the software DEFORM for simulation analysis. The finite element model of CWR is demonstrated in Fig. 1. In this paper, the model is divided via the absolute grid method, and the smallest unit size is set to $1.5 \mathrm{~mm}$ and the maximum size of the unit is set to $3.0 \mathrm{~mm}$. Billet material is AISI 1045. The original billet diameter $\mathrm{D}$ is $40 \mathrm{~mm}$, and the roller diameter is $500 \mathrm{~mm}$. In addition, the initial temperature for workpiece is $1150^{\circ} \mathrm{C}$. Coulomb friction is used to simulate the contact, and the friction coefficient is 0.2 .

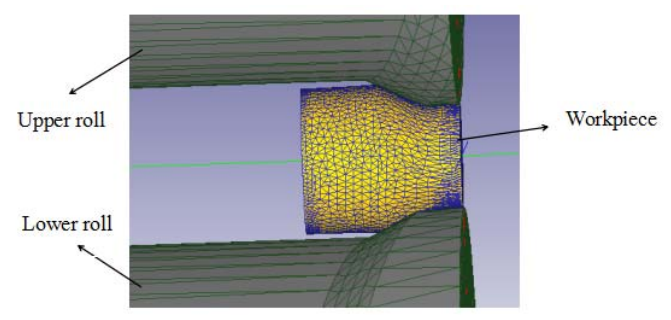

Fig. 1 Finite element model

\subsection{The calculation method of the volume of stub bar}

After research and analysis, center offset distance is generally less than $0.2 \mathrm{~mm}$ after rolling. Therefore, in order to calculate the end volume of each of the conditions accurately, we select the center point of the cross-section of the blank and use it as the center of the circle with a radius of $0.1 \mathrm{~mm}$ and $0.2 \mathrm{~mm}$, take 12 evenly spaced nodes respectively on the two circles. After rolling, the length of the non-concavity on one side of the workpiece is the average value of the axial coordinate of 25 nodes (the axial coordinate of symmetric center of the workpiece is 0 ). As shown in Fig.2. The formula of the volume of the end as follows:

$$
\mathrm{V}=\frac{\pi}{4} d_{0}^{2} l-\frac{\pi}{50} d_{1}^{2} \cdot \sum_{i=0}^{24} p_{i}
$$

Where: $\mathrm{V}$ is end volume $\left(\mathrm{mm}^{3}\right), d_{0}$ is material diameter $(\mathrm{mm}), l$ is the length of original blank $(\mathrm{mm}), d_{1}$ is diameter after rolling $(\mathrm{mm}), p_{i}$ is the axis coordinate of the $i$ points $(\mathrm{mm})$. 


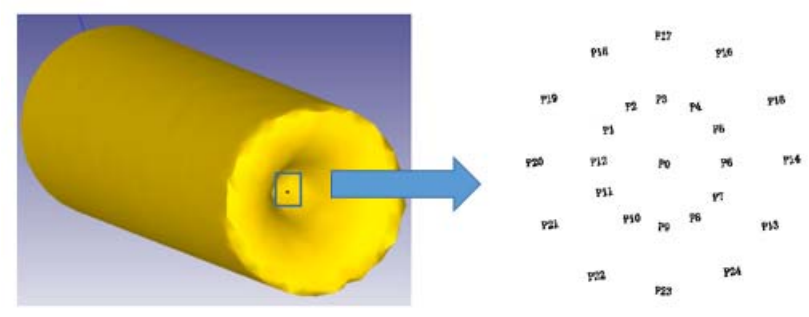

Fig.2. The locations of points on the end of blank

\section{Blank Compensation Method}

Due to the extrusion of the surface of the metal to the center of the metal, the external metal flows faster than the internal metal in the generating process of the concavity $[3,4,5]$, which will result in cavity in the middle of the blank end. This hole just reflects the uncoordinated material volume caused by fast flow of the outside material. If when the cavity begin to appear, the external material was reduced appropriately. And this reduction is in conformity with the redundant external material caused by fast flow of the outside material, the missing volume in the middle part of the material caused by the flow non-uniformity is compensated to avoid cavity, this is the principle of the blank compensation.

To analyze the effect of critical process parameters on the volume of the end under the circulararc face, the paper design blank shape as shown in Figure 3, the central angle $\theta$ shown in Figure 3, too.

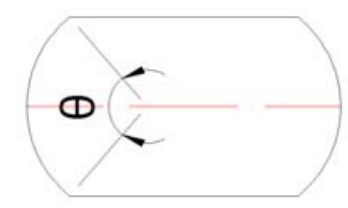

Fig.3 Blank section view and the central angle of circular-arc end

\section{The Influence of Process Parameter on the Volume of Stub Bar under Two End Shapes}

\subsection{The influence of forming angle on the volume under two end shapes}

Fig. IV illustrates the effect of forming angle on the volume of stub bar when the spreading angle is $7^{\circ}$, area reduction is $50 \%$ and mill length is $50 \mathrm{~mm}$. It can be seen from Fig. 4 that the forming angle has a little impact on the volume. With the increase of forming angle, the volume first decreases and then increases. 


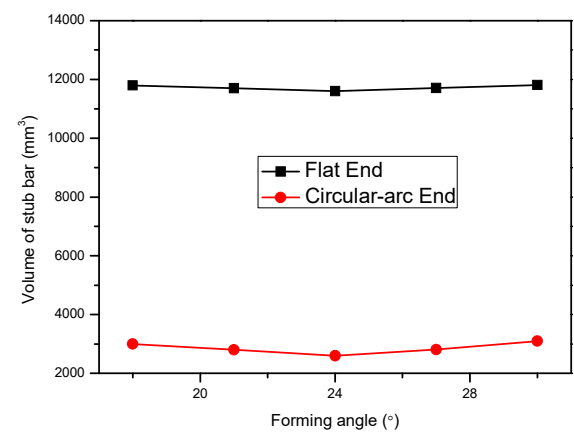

Fig.4 Relationship between forming angle and the volume of stub bar

In the first place, the great the forming angle, the larger the rolling reduction of rolled piece per half a circle in the process of CWR, the smaller the time of the repeated compression in the same place of rolled piece when the workpiece was processed to a predetermined size, the small the inhomogeneity of axial deformation between the surface and the core part of the metal. Finally, the volume of stub bar decreases. On the other hand, with the increase of forming angle, the contact length between the rolled piece and the dies in the rollers in the axial direction of will decrease and the friction constraints in contrast to the metal flow direction will also decrease so that the metal will be easier to deform along the axial direction, that will make the inhomogeneity of axial deformation between the surface and the core part of the metal increased to a certain extent and the volume increased. A result of the combined action of these two aspects is that the forming angle will have a little effect on the volume.

\subsection{The influence of spreading angle on the volume under two end shapes}

In order to get the varying rule of volume of stub bar with the changing of spreading angle when forming angle is $26^{\circ}$, area reduction is $50 \%$ and mill length is $50 \mathrm{~mm}$, the Fig. 5 was created. Closely examining the Fig.5, we found that spreading angle has a significant effect on the volume, and the volume decreases with increasing spreading angle.

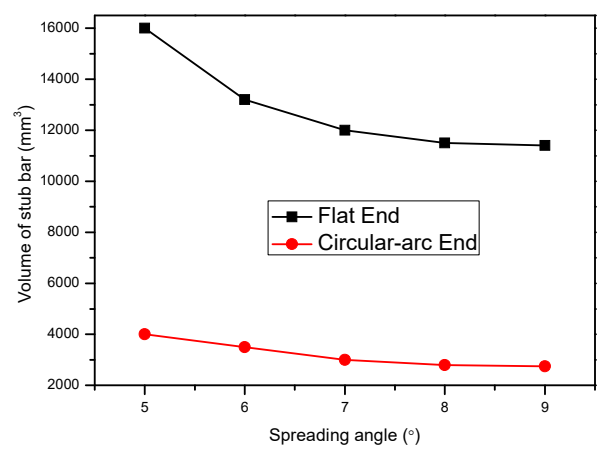

Fig.5 Relationship between spreading angle and the volume of stub bar

With the increase of spreading angle, the instantaneous broadening amount and rolling reduction of rolled piece increase per half a circle, and the contact length between rollers and workpiece along the axis direction increases so that the friction force contrast to the metal flow in axial direction increases. Subsequently, the axial rolling force decreases, the axial metal flow is difficult and the volume decreases. 


\subsection{The influence of area reduction on the volume under two end shapes}

Fig. 6 is the effect of area reduction on the volume of stub bar when the forming angle is $26^{\circ}$, spreading angle is $7^{\circ}$ and area reduction is $50 \mathrm{~mm}$. It can be seen that the volume increases first and then decreases with the increase of area reduction.

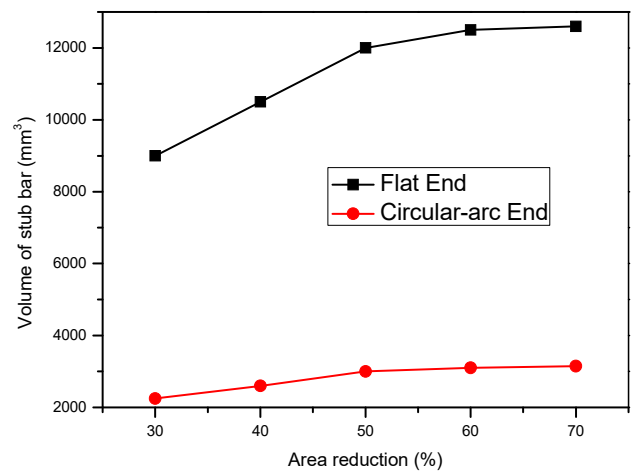

Fig.6 Relationship between area reduction and the volume of stub bar

As seen from the Fig.6, when the area reduction varies in a certain range, the deformation is mainly concentrated on the surface of workpiece in the process of rolling, and the uneven degree of the axial deformation between the rolling surface and the central portion of the metal will increase due to the gradual accumulation, so the volume will increase. When the area reduction increases to a certain degree, the deformation will penetrate into the center of workpiece, the difference of axial metal flow between the surface and core of workpiece becomes small. On the other hand, the contact area of friction force also increases so that the axial metal flow becomes difficult owing to the function of great friction on the workpiece surface. Hence, the volume of stub bar will have a tendency to decrease when the area reduction increases to a certain value.

\subsection{The influence of mill length on the volume under two end shapes}

It can be observed from Fig.7 that increasing the mill length increases the volume when mill length is less than $60 \mathrm{~mm}$ and keeps unchanged approximately when mill length is more than $60 \mathrm{~mm}$, where the forming angle is $26^{\circ}$, spreading angle is $7^{\circ}$ and area reduction is $50 \%$.

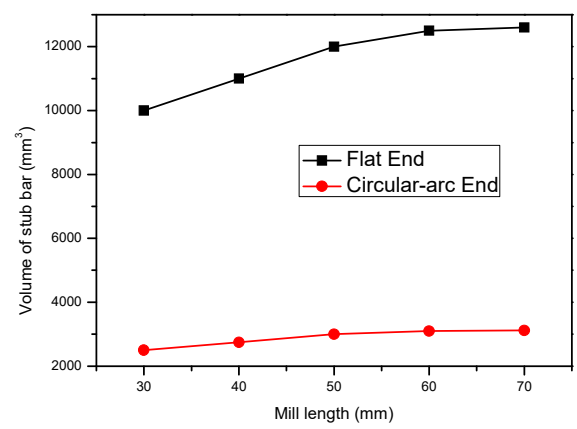

Fig.7 Relationship between mill length and the volume of stub bar

When the mill length is less than $60 \mathrm{~mm}$, the inhomogeneity of axial deformation between the surface and the core of the metal intensifies gradually with the increasing of mill length. However, when mill length exceeds a certain extent, due to the restriction of undeformed end to the deformed 
surface metal at the beginning of the rolling, there is no concavity generation at this time and the volume is unchanged approximately.

\section{Experimental Verification}

For the purpose of further validating the correctness of using the circular-arc end blank to reduce or even eliminate the concavity, as shown in Fig.8, the experiment under two end shapes was carried out through the D46-50×400 type rolling mill. The blanks under flat and circular-arc end are clearly reflected in Fig. 9 and 10, respectively. With regard to the symmetry, here only one side of the blank is processed into the arc shape. The experimental parameters are listed in Tab.1.

Tab.1 Experimental scheme

\begin{tabular}{c|c|c}
\hline Items & $\begin{array}{c}\text { Mill length } \\
\mathrm{L} / \mathrm{mm}\end{array}$ & $\begin{array}{c}\text { Area reduction } \\
\psi / \%\end{array}$ \\
\hline 1 & 30 & 50 \\
\hline 2 & 40 & 50 \\
\hline 3 & 50 & 40 \\
\hline 4 & 50 & 50 \\
\hline 5 & 60 & 60 \\
\hline
\end{tabular}

After rolling, the rolled pieces were disposed by fast wire cutting processing in order to facilitate the measurement of concavity depth of the workpiece, and the workpieces after wire cutting treatment under flat end and circular-arc end are represented in Fig.11 and 12, respectively. To avoid the manual measurement error as far as possible, multimetering was used to gain the length of the non-concavity for each of the rolled pieces. Subsequently, the average is put into the formular (I) as the final volume of stub bar.

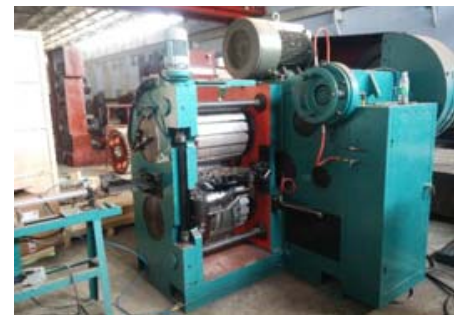

Fig.8 Experimental equipment

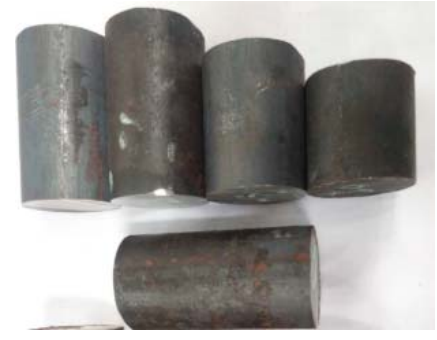

Fig. 9 Flat billets

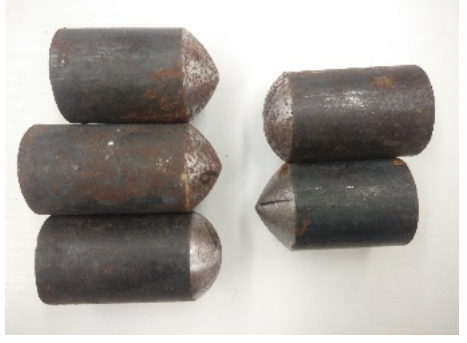

Fig. 10 Circular-arc billets

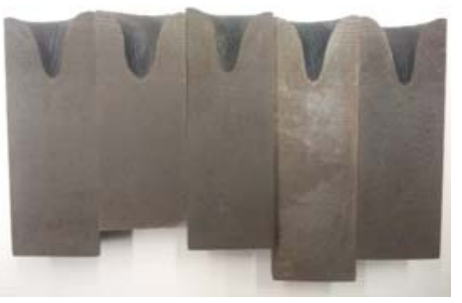

Fig.11 Flat end workpieces after linear cutting 


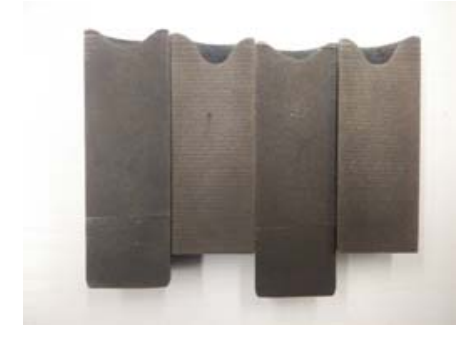

Fig.12 Circular-arc end workpieces after linear cutting

It can be seen from Fig. 11 and Fig.12 that the volume under the circular-arc end is significantly less than the flat end. The average of the flat end volume is $11980 \mathrm{~mm} 3$, which is 4.17 times of the circular-arc end volume $\left(2875 \mathrm{~mm}^{3}\right)$, it is proved that the method is effective to control the concavity.

\section{Conclusions}

1) The impact of process parameters on the end volume of the workpiece under two end shapes was analyzed by means of finite element simulation and theoretical analysis, and the volume under the circular-arc end is significantly less than the flat end. In the end, the feasibility of using circulararc billet to reduce the concavity were verified by rolling experiment.

2) The volume of stub bar is in direct proportion to the spreading angle, and decreases at first and then increases with the increasing of the forming angle, and increases at first and then decreases with increasing of the area reduction, and increases at first and then keep approximately invariant with increasing of mill length.

\section{References}

[1] MA Zhenhai, YANG Cuiping, HU Zhenhuan. Study on effect factors of end concavity in cross wedge rolling[J]. Forging Stamping Technology, 2002, 27(1):29-31.

[2] ZHANG Xiaoying. Simulation of deformation principle of near net shape cross wedge rolling process to form shafts without concavitys[J]. Forging Stamping Technology, 2013,2 (38) :156-160.

[3] WEI Xinhong, SHU Xuedao. Effect of end shape of blank on cavity of workpiece with CWR[J]. Hot Working Technology, 2011,40(21):61-63.

[4] LIU Chuan, SHU Xuedao. Effect of circular are angle on end concavity of CWR rolled piece[J]. Hot Working Technology, 2015,44(15):101-108.

[5] YANG Guang, ZHANG Kangsheng, DUAN Wanze, et al. Effect of process parameters on the end scrap of no-step--end workpieces by cross wedge rolling[J]. Journal of University Science and Technology Beijing, 2014,36(7):959-965. 